\title{
Florid Cemento-osseous Dysplasia: A Report of Two Cases and Literature Review
}

\author{
Prashanth Panta ${ }^{1}$, Imran Shahid ${ }^{2}$, Shankargouda Patil ${ }^{3}$, Mukund Seshadri ${ }^{4}$
}

\begin{abstract}
Aim: This report aims to provide the clinical and radiographic features of two symptomatic Indian patients with florid cemento-osseous dysplasia (FCOD), along with a discussion of the differential diagnosis, potential challenges, and therapeutic implications.

Background: FCOD is a rare, multifocal, periapical, and bilateral condition involving the premolar and molar region of the posterior mandible and sometimes the maxilla.

Case description: The first patient is a 30-year-old female with a recent history of dental pain. The patient was otherwise healthy and the medical history was unremarkable. The second patient is a 50-year-old female with a history of orthodontic therapy. Radiographic evaluation using cone-beam computed tomography (CBCT) revealed bilateral involvement of the posterior mandible, sparing the entire maxilla in both patients Clinical significance: For a pathognomonic condition like FCOD, a radiology survey alone is often sufficient to arrive at the final diagnosis, and therefore surgical interventions should ideally be avoided.

Keywords: Bone remodeling, Florid cemento-osseous dysplasia, Mandible, Orthodontic treatment, Osteomyelitis, Secondary infection. The Journal of Contemporary Dental Practice (2021): 10.5005/jp-journals-10024-3029
\end{abstract}

\section{INTRODUCTION}

Florid cemento-osseous dysplasia (FCOD) is a multifocal disease that often presents as bilateral bone pathologies in middle-aged individuals and predominantly affects females. FCOD is also referred to as "gigantiform cementomas" and "familial multiple cementomas". " FCOD is often restricted to the premolar-molar region of the mandible and occasionally the maxilla. FCOD is characterized by the replacement of healthy jaw bone by avascular masses of cementum and fibrous tissue. The earliest case series on FCOD was reported by Waldron et al. and Melrose et al. and were considered to be reactive fibro-osseous jaw lesions of periodontal origin. ${ }^{1-3}$

FCOD is relatively rare and is often discovered as an incidental finding on routine radiographic surveys. Although FCOD is usually asymptomatic, the pain was reported as a presenting symptom in some patients. ${ }^{1}$ Individual lesion in FCOD can vary in size and can appear as diffuse cloud-like masses at the apices of premolar-molar (mandibular) teeth, primarily composed of cementum like material. Patients may also develop cortical expansion, jaw perforations, and some even develop osteomyelitis, an underestimated complication of FCOD. ${ }^{4}$ In this report, we describe the clinical presentation of two female patients from the Indian sub-continent, along with their radiologic features on cone-beam computed tomography (CBCT) examination.

\section{CASE 1}

A 30-year-old female reported to a private dental office with complaints of pain and occasional swelling in the left-back tooth region for 15 days. The patient was otherwise healthy and the medical history was unremarkable. Clinical oral examination revealed several grossly destroyed permanent teeth and advised a panoramic radiograph. Based on the radiograph findings, the

\begin{abstract}
1,2 Department of Oral Medicine and Radiology, MNR Dental College and Hospital, Sangareddy, Telangana, India

${ }^{3}$ Department of Maxillofacial Surgery and Diagnostic Sciences, Division of Oral Pathology, College of Dentistry, Jazan University, Jazan, Kingdom of Saudi Arabia

${ }^{4}$ Department of Oral Oncology, Roswell Park Comprehensive Cancer Center, Buffalo, New York, United States

Corresponding Author: Prashanth Panta, Department of Oral Medicine and Radiology, MNR Dental College and Hospital, Sangareddy, Telangana, India, Phone: +91-9701806830, e-mail: maithreya.prashanth@gmail.com

How to cite this article: Panta P, Shahid I, Patil S, et al. Florid Cementoosseous Dysplasia: A Report of Two Cases and Literature Review. J Contemp Dent Pract 2021;22(3):304-309.
\end{abstract}

Source of support: Nil

Conflict of interest: None

surgeon considered a diagnosis of "osteomyelitis" and planned a surgical procedure to remove the abnormality. For a second opinion, she visited the Oral Medicine Department at the MNR Dental College and Hospital. Multiple radiopacities in the right premolar-molar region of the mandible (\#44, \#45, \#46, \#47, and \#48) and poorly mineralized radiopacities on the left side along with a peculiar derangement in the trabecular pattern were observed in our initial radiological surveys (Fig. 1). There was no clinically noticeable jaw swelling. CBCT imaging revealed a distinct radiolucent halo around each of the individual radiopacities (Fig. 2A). The axial sections in CBCT (Fig. 2) demonstrated proximity of radiopaque lesions with the root tips of right and left premolar and molar mandibular teeth (Figs. 1 and 2B), and axial and reconstructed view showed numerous perforations and protuberances within the affected mandible (Fig. 2C). The radiographic and clinical findings 


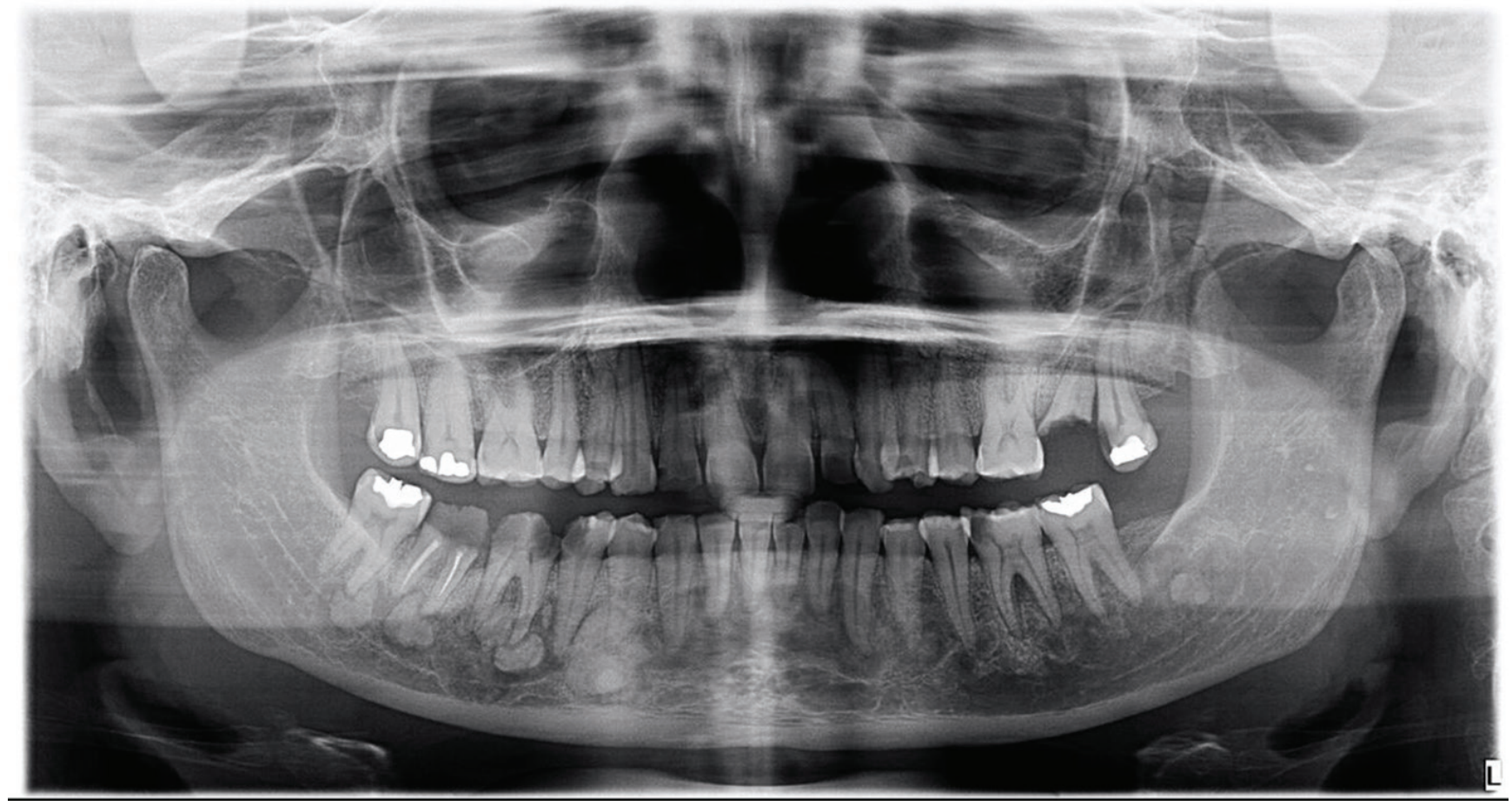

Fig. 1: Panoramic radiograph of case 1 shows multiple periapical lesions in the lower jaw with disturbed trabecular architecture and cystic cavities on the left side
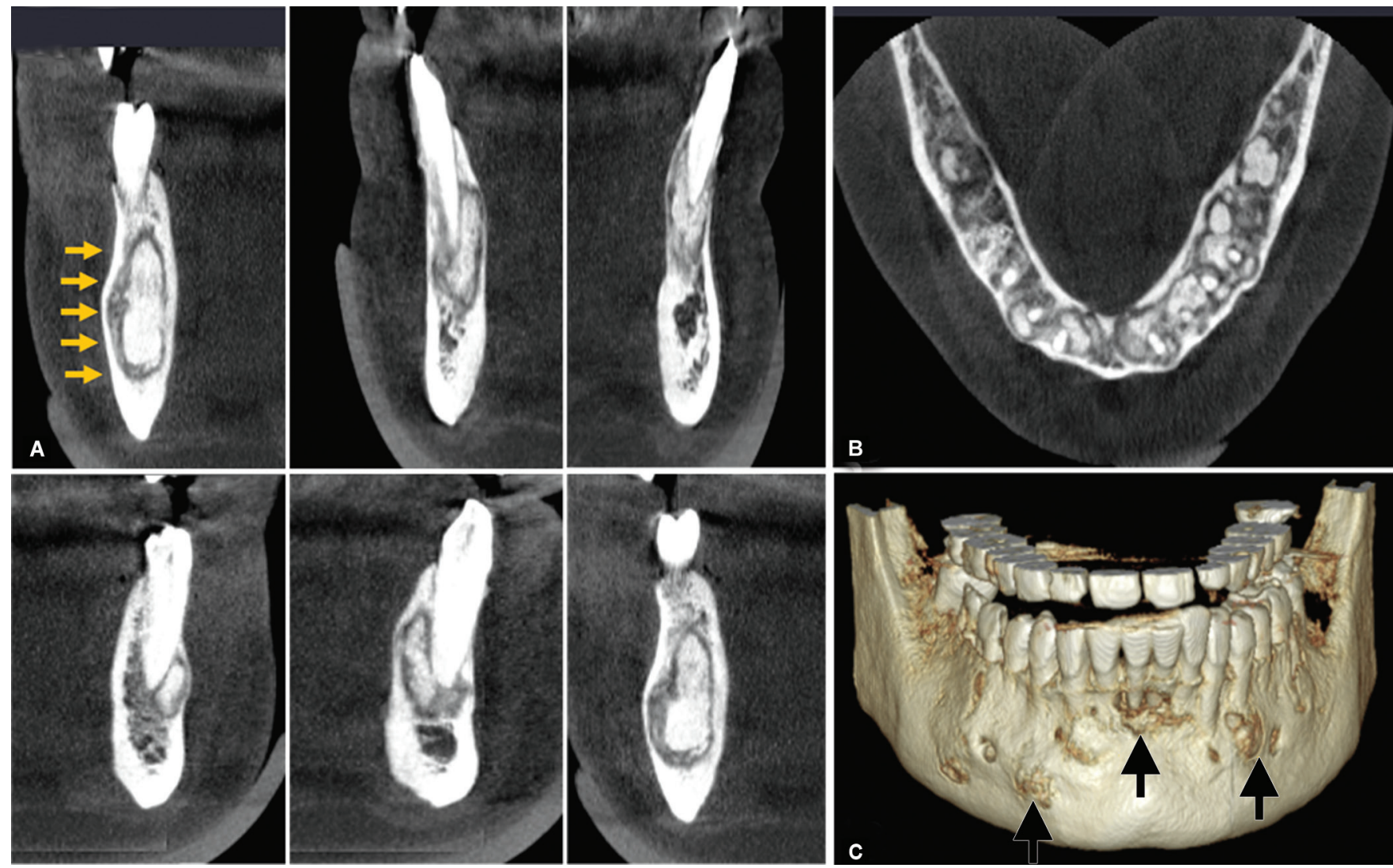

Figs 2A to C: (A) Periapical lesions on coronal sections show a mixed appearance, surrounded by a radiolucent rim; (B) Periapical mixed lesions on axial sections show numerous radio-opaque masses close to tooth apices; $(C)$ The reconstructed 3D view of case 1 shows numerous perforations (black arrows) along with bony protuberances 
together suggested a final diagnosis of FCOD. The patient was empirically managed with analgesics and antibiotics; root canal treatment was performed for \#36, \#45, \#46, and dental crowns were placed for root canal treated \#36, \#45, and \#46 also for previously treated \#47. Although surgical extraction was advised for grossly decayed \#27, she did not undergo any treatment. All lower teeth were endodontically managed, but subsequent follow-up with the patient was not successful. We have notified the patient about her condition and advised periodic dental screening and good oral hygiene.

\section{CASE 2}

A 50-year-old female patient visited the Oral Medicine Department at MNR Dental College and Hospital with moderate pain in the left lower back tooth region for 1 week. The pain was continuous, nonradiating, and localized, and specifically aggravated on the intake of cold foods. She reported the first onset of pain 3 years back; she mentioned that pain used to subside following the administration of analgesics but recurred again after a few weeks. Past dental history revealed previous orthodontic treatment (20 years ago), for which therapeutic extraction of premolars was conducted (missing \#14, \#24, \#34,\#44). Radiographic investigations included panoramic radiographs (Fig. 3) and CBCT imaging (Fig. 4). Welldefined radiopaque lesions with a radiolucent halo in left (\#35,\#36, and \#37) and right quadrants (\#45, \#46 mesial, and distal root tip) of the mandible were detected on radiologic examination. The left and right maxilla was completely spared. Periapical radiographs had shown evidence of proximal secondary caries around a dental restoration, and the dental abscess was mainly localized to \#35 and mesial root of \#36, which was well defined and rounded. A radiopaque mass (cementacle) was observed within the dental abscess in the \#35 region. A diagnosis of an acute dental abscess with \#35 and \#36 with concurrent FCOD was considered. An empirical prescription of analgesics and broad-spectrum antibiotics was advised, followed by root canal treatment in \#35, which the patient underwent. However, we could not evaluate the patient following treatment. We notified her about her condition and advised periodic dental screening and good oral hygiene.

\section{Discussion}

In the present report, we describe the clinical presentation and radiologic features of two Indian female patients in their third and fifth decade with concurrent pulpal pathology. In the first patient, there was radiological evidence of pulpal involvement in decayed \#36. Hence, both our reports do not represent symptomatic FCOD cases that present with intermittent pain. ${ }^{1}$ Both the reported patients represent the coexistence of concurrent pulpal infection in FCOD bone. Although coincidental, this highlights a paramount concern related to possible secondary infection sources (pulpperiapical) of hypo-vascular FCOD bone. The presence of periapical infection and ideal endodontic management protocols for carious teeth in FCOD patients has not been widely reported. We advised endodontic rehabilitation of carious and grossly destroyed teeth and recommended simple oral hygiene practices to avoid subsequent dental caries and periodontal issues in the future. We have cautioned our first patient not to undergo any excessive surgical treatment explaining the osteomyelitis complications connected with overenthusiastic surgery. As FCOD shows a high potential for misdiagnosis and unnecessary intervention (as observed in case 1) clinicians must bear adequate knowledge of this condition..$^{4-7}$ Interestingly, the periapical lesions on the left and right quadrants were found at different development stages and were surrounded by a radiolucent halo. Since FCOD is chiefly a radiographic diagnosis, it is critical to understand its differential

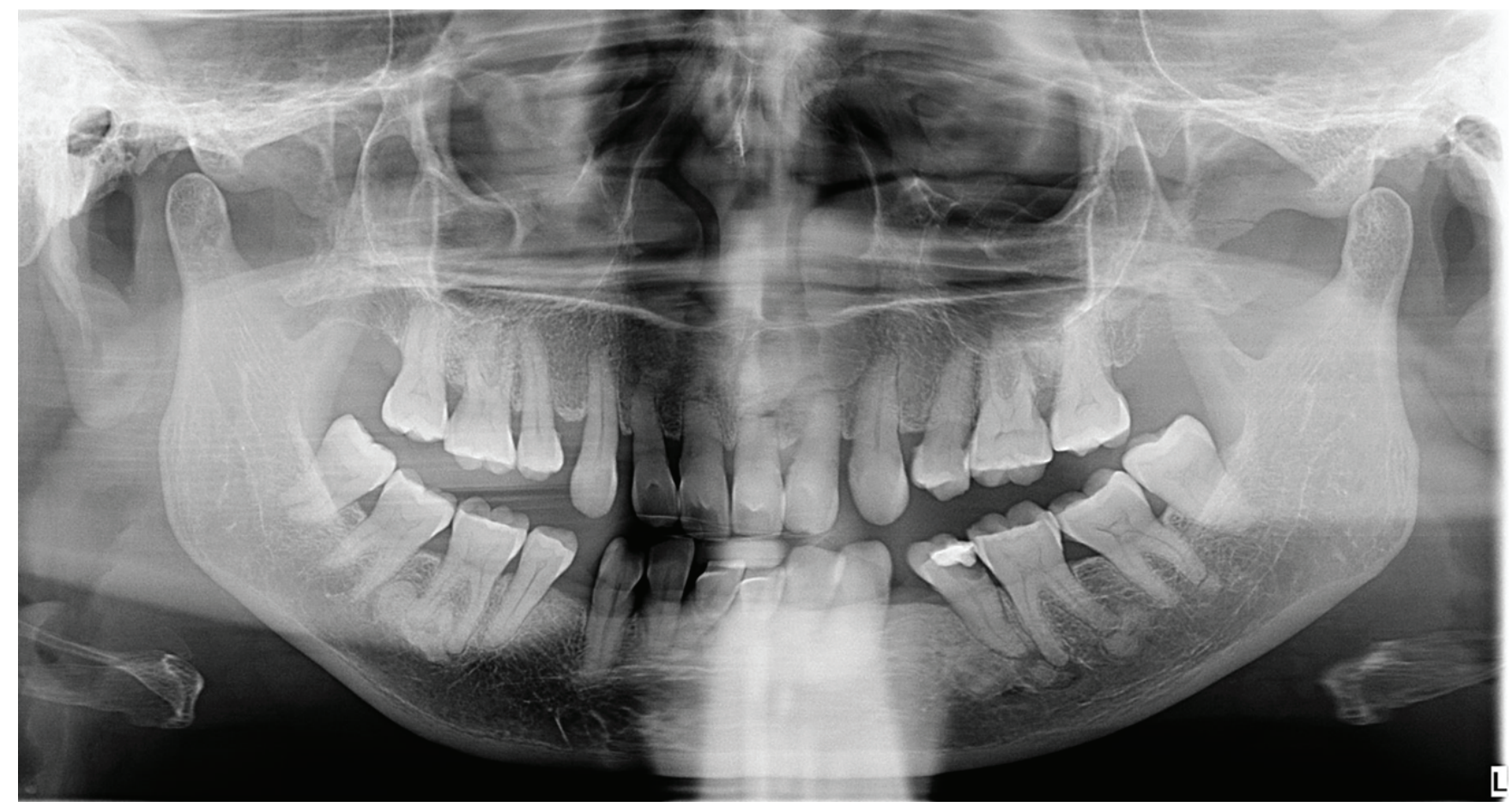

Fig. 3: Panoramic radiograph of case 2 shows multiple periapical lesions in the lower jaw localized to the molar region on the right mandible and premolar-molar region on the left mandible. Also, first, premolars are missing due to prior orthodontic therapy 

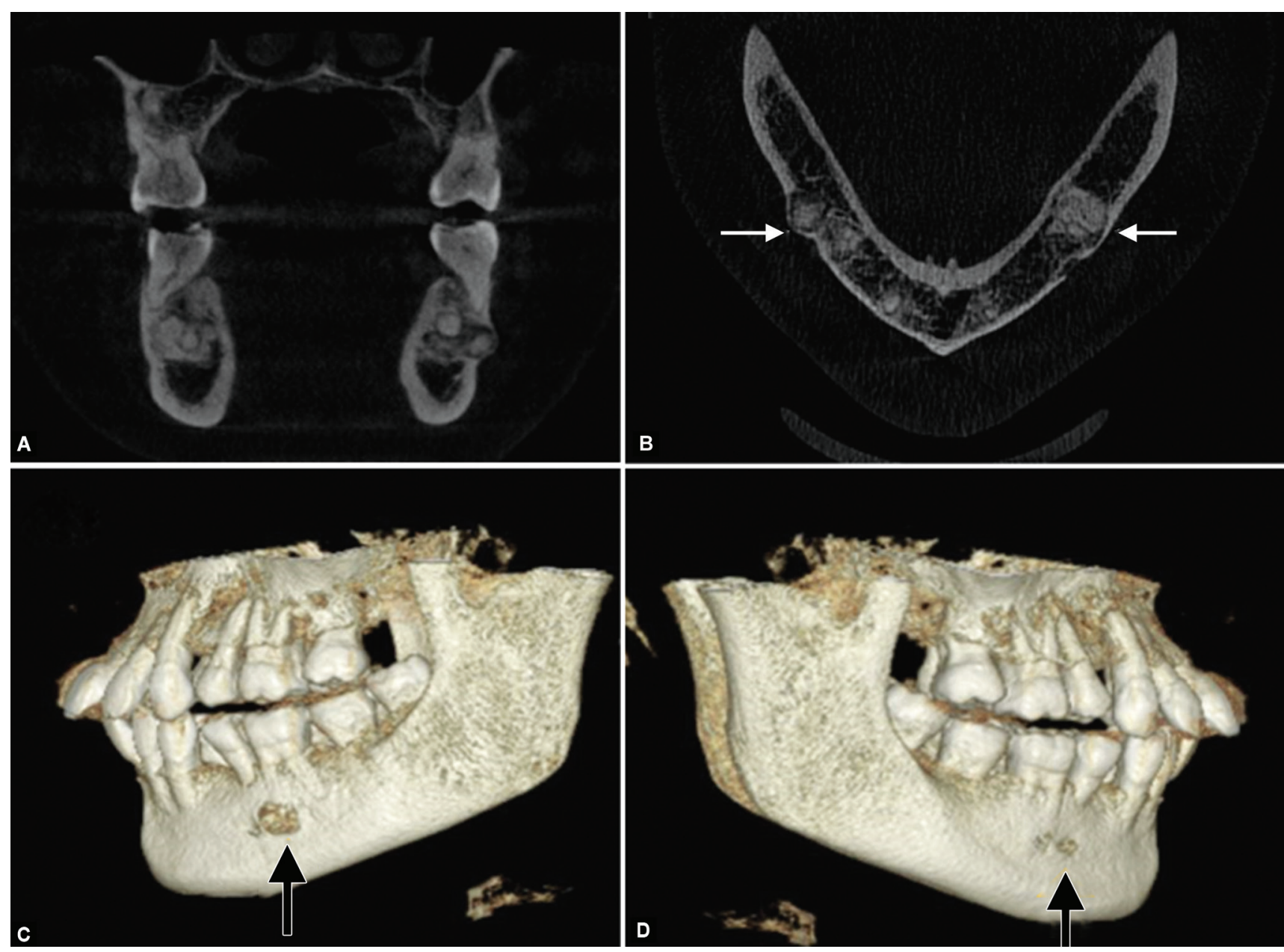

Figs 4A to D: (A) Periapical lesions on coronal sections show a mixed appearance, surrounded by a radiolucent rim; (B) Periapical mixed lesions on axial sections show numerous radio-opaque masses close to tooth apices; (C, D) Reconstructed 3D view of case 2 shows severe maxillary protrusion and two bony-perforations (black arrows) at the premolar-molar region of the left and right mandible

diagnosis. The differential diagnosis of FCOD must include the spectrum of conditions that present as diffuse, generalized radiopacities and that involve the periapical, tooth-bearing region of jaws. FCOD belongs to the class of cemento-osseous dysplasias (CODs) that can be categorized into three parts: (i) periapical cemento-osseous dysplasia (PCOD), (ii) focal cemento-osseous dysplasia (Focal COD), and (iii) florid cemento-osseous dysplasia (FCOD). CODs are considered "reactive lesions arising in toothbearing regions", as postulated by Waldron in $1993 .{ }^{8}$ Since these conditions were closely associated with the tooth-bearing portion of jaws, they were considered periodontal ligament in origin. The frequent occurrence of FCOD in the dentulous regions of jawbones (especially the premolar-molar region) further substantiates the possible role of trauma-induced due to masticatory loading.

Fibro-osseous conditions like fibrous dysplasia (FD) are more common and frequently present as an expansive growth, especially involving the buccal aspect. Unlike FD, FCOD displays changes mainly localized to the periapical bone. Developmentally, FCOD progresses from an initially radiolucent stage (composed primarily of fibrous tissue) to a mixed (composed of cementum like material in a swirling pattern) and later to a radiopaque stage. ${ }^{8}$ Unlike FCOD, PCOD is encountered in the anterior mandible (i.e., incisor region). PCOD also demonstrates a marked female predilection
$(\mathrm{F}: \mathrm{M}=9: 1)$, and the lamina dura is often lost in the adjacent teeth. ${ }^{8}$ It is often bilateral and multiple similar to FCOD, but can rarely be solitary. ${ }^{8}$ PCOD lesions are associated with vital pulps, do not cause any detectable cortical plate expansion, and may cause hypercementosis of affected teeth, and very rarely causes tooth resorption. ${ }^{8}$ In contrast, larger PCOD lesions may cause a jaw expansion in the undulating configuration. ${ }^{8}$ Interestingly, PCOD lesions seem more often among Asians, similar to FCOD. Focal COD is seen with single posterior teeth in the premolar-molar region, nearly always unilateral, and is seen more often in younger individuals, with a likelihood to increase in size. ${ }^{8}$ FCOD, in contrast, is a more generalized pathological event of jaws, with typical multiquadrant involvement as mentioned previously. The interiority in FCOD shows a cotton wool appearance and involves at least two quadrants of the mandible, as a hallmark finding.

Diffuse sclerosing osteomyelitis (DSO) also shares a similar radiological picture of FCOD but is often associated with infectious foci and therefore seen on one side that harbors the decayed tooth. DSO may be included in the differential diagnosis in case 2 (associated with infected pulps in \#35); however, the striking bilateral distribution of lesions suggests preexisting FCOD, and concurrent periapical changes seem to be supraadded. However, perforation with \#35 highlights the preexisting cementum like 
mass, as dental abscesses rarely perforate the jaws. Also, there was no draining sinus in the oral cavity adjacent to the affected teeth. Periapical abscess in \#35 is rounded, and the "cementum like masses" associated with the mesial root of \#36 seems to be enclosed into the periapical lesion. Similarly, in case 1, decay is noticeable in \#36 along with pulpal involvement, periapical widening, and interdental bone loss. Paget's disease (PD) is characterized by increased osteoblastic activity and closely mimics the radiological features of FCOD, but is a polyostotic skeletal disorder involving multiple skeletal bones (e.g., pelvis, femur), skull, and less frequently the jaws. Also, the pagetoid bones increase several-fold in thickness/volume, and the maxilla is more frequently involved over the mandible. ${ }^{8}$ It occurs predominantly in males belonging to the older age group (6th decade). Moreover, bone modeling in PD is too dynamic compared to FCOD bones. If jaw feature suggests PD, clinicians must rule out the involvement of other skeletal bones through metabolic imaging (SPECT/CT). They can also screen for elevation in serum alkaline phosphatase, which is highest in PD than any other pathological condition, especially during the disease's osteoblastic phase. In contrast, elevation in serum alkaline phosphatase is rare with monostotic conditions like FD or FCOD. Another biomarker "hydroxyproline", a collagen metabolite, is often noticed in the urine of PD patients. ${ }^{8}$

Individual radiopacities in FCOD demonstrate a radiographic rim (similar to the capsule in odontoma and cementoblastoma), which is unlikely in metabolic conditions like PD or infective conditions like DSO; this feature is unique to the benign tumors and fibro-osseous tumors of the mandible. The co-occurrence of simple/idiopathic bone cyst (IBC) and FCOD have been reported in the literature, first confirmed by Melrose et al. in $41 \%$ of 34 reported cases. ${ }^{3,9,10}$ In a report on 20 IBC cases, 30\% were associated with FCOD.10 The presence of IBC has been underestimated in FCOD and COD literature and was frequently cited as a rare occurrence. The presence of bone cysts in FCOD can result in low-grade intermittent pain, which clinicians need to consider.

The characteristic periapical radiolucencies of FCOD can be misinterpreted by clinicians, who may attempt therapy suspecting it to be of endodontic origin. ${ }^{5,6}$ In such a scenario, it is easy to exclude endodontic origin through pulp-vitality investigation. A tooth with a periapical lesion arising from endodontic/pulpal-periapical infection is generally non-vital on vitality testing.

Oral health clinicians/dental surgeons must bear a good understanding of periapical opacities and their corresponding differential diagnosis. CBCT is an excellent method to assess challenging presentations. This modality can provide precise details concerning the precise relationship between the lesion and adjacent teeth, internal structure, surrounding radiolucent rim, and finer details concerning cortical plate expansion and perforation. Interestingly, CBCT imaging in the present report revealed a mild form of bilateral cortical plate expansion and perforation at numerous locations in both cases. In some case reports, FCOD may be associated with sensory nerve disturbances like "paresthesia", ${ }^{11}$ which is often associated with jaw malignancies and chronic infections like osteomyelitis. In one of the case reports, an FCOD patient presented with swelling and tenderness in the mandibular posterior region ${ }^{12}$, mimicking periapical, inflammatory jaw conditions. Such symptoms could be due to osteomyelitis or periapical abscess supraadded to preexisting FCOD, as in the present case.
Concurrent FCOD along with type-I neurofibromatosis and peripheral giant cell granuloma has been previously reported. ${ }^{13}$ Similarly, an association between FCOD and the dentigerous cyst has also been reported. ${ }^{14}$ To our knowledge, there is only one report of malignancy (malignant spindle cell tumor) arising in the mandible of an FCOD patient. ${ }^{15}$ Both our patients were free of any such associations and have displayed no syndrome abnormalities.

Clinicians must advocate great caution, and it is best if longterm follow-ups are conducted in FCOD cases. Also, immediate restoration of teeth at the early stages of dental caries (enamel and dentinal caries) may avoid periapical complications like osteomyelitis. Tooth removal in patients with FCOD may lead to osteonecrosis due to hypovascularity of cemental masses, and hence follow up is needed. Sequential radiographs are useful as one could access the growth potential of this lesion. FCOD is likely to be infected if dental decay is neglected, as in case 1. In such cases, antibiotics may be required. FCOD could also co-exist with osteomyelitis, as reported in a few cases. ${ }^{16,17}$ In such cases, careful sequestrectomy can be performed, and good antibiotic coverage is required to minimize the dissemination of bacterial infection through the avascular tissues (secondary infection). ${ }^{16,17}$

Although numerous familial cases of FCOD have been reported, this condition's genetic basis remains elusive. However, mutations in the ANO5 gene were identified in a Chinese family with Familial FCOD. ${ }^{18}$ Mutations in ANO5 were previously identified in gnathodiaphyseal dysplasia, an autosomal dominant syndrome characterized by cemento-osseous dysplasia of jawbone, bone fragility, and diaphyseal sclerosis in tubular bones. ${ }^{19}$ Since FCOD is mainly asymptomatic and familial occurrences are known, it is vital to screen radiological confirmed FCOD cases and immediate family members for a mutation in ANO5. Inadequate osteoblast differentiation resulting from ANO5 mutations ${ }^{19}$ and masticatory loading may lead to disorganized bone remodeling and avascular cementoid formations in FCOD patients.

The literature is also contraindicating orthodontics in FCOD due to the need for additional extractions. Some authors have also emphasized that affected bone may not undergo efficient remodeling, but this is still debatable, and FCOD case reports summarizing the effect of orthodontic treatment could be beneficial. ${ }^{20}$ An interesting observation in case 2 is the previous history of orthodontic treatment performed nearly 20 years ago. Since previous radiographs are lacking, it may be possible that orthodontics forces may have resulted in FCOD, or this lesion may have developed irrespective of orthodontic treatment? To the best of our knowledge, there is no report on the emergence of FCOD following orthodontic intervention. In the literature, only two reports show the result of orthodontic treatment in known FCOD patients. ${ }^{21,22}$ In such patients, as suggested by Minhas et al., a non-extraction-based orthodontic approach may be chosen. ${ }^{21}$ In the literature, there is a noteworthy report of a 24-year-old female FCOD patient who was under surveillance for 13 years following orthodontic therapy. ${ }^{23}$ The success rate of orthodontics may be higher in patients with predominant radiolucent component than in those with highly mineralized radiopaque lesions; if orthodontic treatment is required, it may be better to initiate at early radiolucent stages itself. ${ }^{20,21}$ Another vital question is concerning the level of tolerance of FCOD bone to implant placement. Although successful implant placement in an FCOD patient has been recently reported ${ }^{24}$, the poorly 
vascularized bone warrants careful evaluation and selection of FCOD patients for implants.

\section{Conclusion}

This report urges all clinicians to carefully pursue the diagnosis of bilateral periapical lesions before initiating surgical treatment. Periodic follow-up of known FCOD cases is recommended as cystic degeneration or malignant transformation may rarely occur. Symptomatic cases can be initiated on analgesics and antibiotics to reduce the possibility of osteomyelitis. Orthodontic treatment should be instituted following a non-surgical approach. Developing models of FCOD is a useful strategy for the development of a more conservative treatment plan. As surgical management is of limited value (due to osteomyelitis complication), clinicians and researchers must study potential pharmaceutical agents that could arrest or reverse the disease process. Clinicians are advised to evaluate the patient's close family members through routine panoramic radiographs to rule out familial occurrences of FCOD.

\section{References}

1. MacDonald-Jankowski DS. Florid cemento-osseous dysplasia: a systematic review. Dentomaxillofac Radiol 2003;32(3):141-149. DOI $10.1259 / \mathrm{dmfr} / 32988764$.

2. Waldron CA, Giansanti JS, Browand BC. Sclerotic cemental masses of the jaws (so called chronic sclerosingosteomyelitis, sclerosing osteitis, multiple enostosis, and gigantiform cementoma). Oral Surg Oral Med Oral Pathol 1975;39(4):590-604. DOI: 10.1016/00304220(75)90201-7.

3. Melrose RJ, Abrams AM, Mills BG. Florid osseous dysplasia. A clinicalpathologic study of thirty-four cases. Oral Surg Oral Med Oral Pathol 1976;41(1):62-82. DOI: 10.1016/0030-4220(76)90254-1.

4. Sarmento DJ, Monteiro BV, de Medeiros AM, et al. Severe florid cemento-osseous dysplasia: a case report treated conservatively and literature review. Oral Maxillofac Surg 2013;17(1):43-46. DOI: 10.1007/ s10006-012-0314-0.

5. Delai D, Bernardi A, Felippe GS, et al. Florid cemento-osseous dysplasia: a case of misdiagnosis. J Endod 2015;41(11):1923-1926. DOI: 10.1016/j.joen.2015.08.016.

6. Huh J-K, Shin SJ. Misdiagnosis of florid cemento-osseous dysplasia leading to unnecessary root canal treatment: a case report. Restor Dent Endod 2013;38(3):160-166. DOI: 10.5395/rde.2013.38.3.160.

7. Krishnan U, Al Maslamani M, Moule AJ. Cone beam CT as an aid to diagnosing mixed radiopaque radiolucent lesions in the mandibular incisor region. BMJ Case Rep 2015;2015:bcr2014207617. DOI: 10.1136/ bcr-2014-207617.

8. Wood NK, Goaz PW. Differential diagnosis of oral and maxillofacial lesions. 5th ed. St. Louis, MO: Mosby; 1997.

9. Martini MZ, Rocha AC, Lemos CA Jr, et al. Fibro-osseous lesions associated with simple bone cysts: three case reports and review of the literature. Minerva Stomatol 2010;59(11-12):671-676.
10. Peacock ME, Krishna R, Gustin JW, et al. Retrospective study on idiopathic bone cavity and its association with cementoosseous dysplasia. Oral Surg Oral Med Oral Pathol Oral Radiol 2015;119(4):e246-e251. DOI: 10.1016/j.0ooo.2014.09.032.

11. Grün $P$, Bandura $P, G$ Gün $A$, et al. Sensory disturbance along the inferior alveolar nerve as a first clinical sign of multiple florid cemento-osseous dysplasia of the mandible: a case report.Int J Surg Case Rep 2018;53:452-457. DOI: 10.1016/j.ijscr.2018.11.036.

12. Grewal HK, Goel P, Batra R, et al. A tender swelling of the left posterior mandible: an unusual case. J Oral Maxillofac Pathol 2014;18(2):332. DOI: 10.4103/0973-029X.140929.

13. Sarmento DJS, Carvalho SHG, JCWPA Filho, et al. Florid cementoosseous dysplasia and peripheral giant cell granuloma in a patient with neurofibromatosis 1. An Bras Dermatol 2017;92:249-252. DOI: 10.1590/abd1806-4841.20175277

14. Sanjai K, Kumarswamy J, Kumar VK, et al. Florid cemento osseous dysplasia in association with dentigerous cyst. J Oral Maxillofac Pathol 2010;14(1):63-68. DOI: 10.4103/0973-029X.72503.

15. Schneider LC, Dolinsky HB, Grodjesk JE, et al. Malignant spindle cell tumor arising in the mandible of a patient with florid osseous dysplasia. Oral Surg Oral Med Oral Pathol Oral Radiol Endod 1999;88(1):69-73. DOI: 10.1016/s1079-2104(99)70195-2.

16. Cavalcante $M B$, de Oliveira Lima $A L$, Júnior $M A$, et al. Florid cemento-osseous dysplasia simultaneous the chronic suppurative osteomyelitis in mandible. J Craniofac Surg 2016;27(8):2173-2176. DOI: $10.1097 /$ SCS.0000000000003095.

17. Singer SR, Mupparapu M, Rinaggio J. Florid cemento-osseous dysplasia and chronic diffuse osteomyelitis. Report of a simultaneous presentation and review of the literature. J Am Dent Assoc 2005;136(7): 927-931. DOI: 10.14219/jada.archive.2005.0294.

18. Lv M, You G, Wang J, et al. Identification of a novel ANO5 missense mutation in a Chinese family with familial florid osseous dysplasia. J Hum Genet 2019;64(7):599-607. DOI: 10.1038/s10038-019-0601-9.

19. Jin L, Liu Y, Sun F, et al. Three novel ANO5 missense mutations in Caucasian and Chinese families and sporadic cases with gnathodiaphyseal dysplasia. Sci Rep 2017;7:40935. DOI: 10.1038/ srep40935.

20. Consolaro A, Paschoal SRB, Ponce JB, et al. Florid cementoosseous dysplasia: a contraindication to orthodontic treatment in compromised areas. Dental Press J Orthod 2018;23(3)26-34. DOI: 10.1590/2177-6709.23.3.026-034.oin.

21. Minhas G, Hodge T, Gill DS. Orthodontic treatment and cementoosseous dysplasia: a case report. J Orthod 2008;35(2):90-95. DOI: 10.1179/146531207225022500.

22. Sethusa MP, Khan MI. The orthodontic management of a patient presenting with cemento-osseous dysplasia. SADJ 2009;64(3):120124. PMID: 19585913.

23. Kato CNAO, Sampaio JDA, Amaral TMP, et al. Oral management of a patient with cemento-osseous dysplasia: a case report. Rev Gaúch Odontol 2019;67:e20190012. DOI: 10.1590/1981-86 372019000123574.

24. Esfahanizadeh N, Yousefi $\mathrm{H}$. Successful implant placement in a case of florid cemento-osseous dysplasia: a case report and literature review. J Oral Implantol 2018;44(4):275-279. DOI: 10.1563/aaidjoi-D-17-00140. 